\title{
Dignity in perioperative practice
}

\author{
Carlos R. Degrandi Oliveira, MD, TSA/SBA, MSc, Lawyer OAB-SP
}

Department of Anesthesiology, Guilherme Álvaro Hospital, Santos, (Brazil)

Correspondence: Carlos R Degrandi Oliveira, Av. Dr Epitácio Pessoa, 131/104 Santos, SP (Brazil) 11045-301; E-mail: degrandi@gmail.com

\section{Abstract}

Any human, healthy, sick, fully conscious or unconscious must be treated with dignity. This is a lesson to be taught to all health professionals, especially those who work in operating rooms and intensive care units. The recognition of human dignity is an important principle in the whole of society, but it is particularly relevant in health, where patients are very vulnerable. Although it may seem easy and natural to behave properly in the presence of a conscious patient, the unconscious or anaesthetized patient should not be expected to give up their rights, beliefs and choices along with their loss of consciousness.

Key words: Dignity, human; Respect; Personhood; Privacy; Perioperative practice.

Citation: Oliveira CRD. Dignity in perioperative practice. Anaesth. pain intensive care 2020;24(5):473-475.

Received: 8 September 2020, Reviewed: - September 2020, Accepted: _ September 2020

Every human individual regardless of gender, race and culture is born with an equal right to respect to maintain dignity. A person strives to be respected and valued throughout his life. Treating with esteem, concern, consideration, being sensitive to cultural differences, privacy and choices, are forms of respect. Also, dignity is defined as being treated with honor, politeness and as equal. Be treated the way any individual would like to be treated. In other words, if a person receives respect, he feels worthy.

The principles of respect and dignity apply to all parts of our daily lives and everyone deserves to be treated in the same way. ${ }^{1}$

The maintenance of dignity in the perioperative period, when the patient may be more vulnerable, is closely linked to awareness of the role of the doctor and other team members, as defenders of ethical issues centered on the rights, choices and beliefs of patients. The creation of an environment that supports and respects human values at the physical and psychological levels throughout the period is paramount and should always be encouraged.

Maintaining the patient's dignity is the obligation of the entire team and one must inspect the other member who does not eventually assume this professional responsibility. It is not uncommon for behaviors that violate patients 'dignity to be reported when professionals disregard patients' concerns, needs and feelings. Many patients report being treated as an object and not a person.

A defender must take positive steps to restore another person's autonomy when he is threatened or diminished, otherwise, any discussion of defense is simply theoretical. The scope of acting as a patient's advocate is a reality because most surgeries require the loss of consciousness from the patient and therefore they are unable to maintain their interests and are particularly vulnerable to abuse and violation of rights. ${ }^{2,3}$

Nursing has always been at the forefront of protecting patients' interests in restoring their health and safety. Advocacy is a fundamental aspect of the ethics of care. The anaesthetized patient who entrusted his life in the hands of the team counts on this care to maintain their dignity and security. ${ }^{2}$

Many of the procedures are potentially invasive and the operating room is scary for most patients, not only 
an unknown place full of highly technical equipment but a place of instruments that lead to injuries and pain.

Whenever possible, a standard pre-anesthetic consultation should be performed, where the first contact with the perioperative clinician is established, when this moment, the patient can express all his doubts regarding the procedure. This contact is a guaranteed right to the patient and allows the anesthesiologist to anticipate, identify potential problems and request evaluation from other specialists when necessary, providing safe planning of the anesthetic act and establishing a relationship of trust and empathy with the patient.

This first contact allows the construction of a relationship with the patient before he arrives at the operating room, which can make a considerable difference. However, the patient will encounter some employees for the first time, and operations department employees should aim to make an immediate positive impression through their approach to the patient.

Infirmary staff should not ask patients to put on gowns earlier than necessary, and patients who go to the gown theatre should wear robes, or be covered. The need to remove underwear is almost negligible, but where necessary, disposable pants can be worn; they can then be cut and removed before surgical preparation.

Patients are very uncomfortable removing their dental prostheses, however, well-adjusted dentures do not need to be removed. The absence of teeth can prevent good ventilation under a face mask. Mobile prostheses can be removed as soon as the patient is unconscious and replaced as soon as the patient awakens. The same goes for eyeglasses and contact lenses. Jewels and props must be removed following institutional protocols. Upon arrival at the perioperative department, the team must be courteous, appropriately approaching the patient, presenting themselves and explaining what will happen. ${ }^{4}$

Patients undergoing surgery are vulnerable to decreased dignity for several reasons. Their bodies are exposed and invaded and, whether they undergo procedures that require general anesthesia, sedation or regional anesthesia, they give control over their bodies and their situation to people who are completely foreign to them. Some who have undergone surgery may have an uncertain diagnosis and outcome and are generally anxious about postoperative pain and recovery. Many have additional vulnerabilities, for example, frailty, having a hearing or visual impairment or other cognitive impairment. Therefore, the perioperative team must understand the circumstances that can contribute to patients' feelings of unworthiness and actively challenge violations perpetrated by colleagues, regardless of status.

Simple actions such as minimizing the patient's exposure during and after positioning and the attitude of respect for the individual and the recognition of their rights and values must be demonstrated by the members of the team involved in the care. They must also be sensitive to the adequacy of their speech, interactions and behavioral attitude. Unnecessary conversations should not be entered into the operating room. If a conversation is to be conducted, it should be done professionally and considering what is said, whether the patient is awake or not.

In preparing the patient, different interventions are performed by the team. They include positioning care, placement of monitoring equipment, among others. In elective procedures, many activities mustn't be performed simultaneously while the patient is conscious, more than one person shouldn't manipulate the patient at the same time, they can do one task at a time, otherwise, it can contribute to negative experiences for the patient. patient. $^{5}$

Patients should be asked how they would like to be treated. This is particularly relevant in the recovery phase, the use of a less used name can make it difficult to respond when trying to wake the patient. The research revealed that a very common practice is to approach patients informally, using their first name, or generically call them 'dear' or 'love'. Most respondents considered this an excessively familiar and disrespectful way to be treated. This is a particularly important point to be considered by the perioperative physician when meeting a patient for the first time. ${ }^{6}$

Anaesthetized patients entrust the theatre team with maintaining their dignity and security. The surgical department staff must take care of the patient's privacy by avoiding any unnecessary exposure and by not allowing extra observers to be present unless the patient has given permission.

It is not uncommon for some theatre staff to make disparaging comments about a patient's appearance, mental health condition or general disposition. This is 
highly disrespectful and every effort must be made to discourage such behavior. Talking about patients violates dignity; patients feel ignored and that they are being treated as an object and not as a person. Communication with patients is crucial. Tutoring and keeping patients informed helps to promote patient dignity. The operational team must be sensitive and discreet. Regardless of whether the patient is awake or fully anaesthetized, maintaining his dignity indicates respect for him as a person, rather than just seeing him as a procedure.

The immediate postoperative phase puts patients in a highly vulnerable situation. They may be in pain, moreover, they may be unable to move or do anything on their own. They are also likely to be seen in an area with multiple patients, with only curtains for privacy. The team must ensure that patients' comfort, safety and dignity are maintained at all times. The recovery professional should be aware that how the patient recovers from anesthesia may not reflect his true personality. When assessing pain, the recovery professional must treat the patient as an individual with individual needs.

It is common practice to use a pain assessment tool. Whichever is chosen, they are seen as a method in which the level of pain is assessed by the patient, and not by the recovery professional, allowing the patient to express his feelings and exercise a certain degree of control over the treatment that receives.

When transferring patient care to the infirmary team, the recovery team must remember to include any new information about the patient's wishes so that the patient's individual needs are considered.

Promoting patient dignity should be a central concern for all health professionals, however, the dignity of patients undergoing surgery is particularly vulnerable. Doctors must be vigilant about the impact of their behavior on patients and be willing to challenge colleagues who compromise patients' dignity. The team must listen and take patient concerns seriously; they must provide assurances that they will take all measures to protect their dignity and they must maintain the trust of their patients by maintaining this guarantee.

The team must recognize the human dignity of all patients in their care and be aware that their interactions will strongly influence the patient's experience. Many patients may require return visits to the operating department, and a single negative experience can result in a detrimental influence on their future treatment decision. ${ }^{7}$

High-quality services that respect people's dignity must show zero tolerance for all forms of abuse, treat each person as an individual, support them in expressing their needs and help them maintain confidence and self-esteem.

\section{Conflict of interest}

None declared by the author

\section{References}

1. All Answers Ltd. Respect and Dignity Basic Right of an Individual [Internet]. November 2018. [Accessed 2 October 2020]; Available from: https://nursinganswers.net/essays/respect-and-dignitybasic-right-of-an-individual-nursing-essay.php?vref=1

2. Marshall C. The concept of advocacy. Br J Theatre Nurs. 1994:4(2):11-13. [PubMed]

3. Freeman M, Pathare S. WHO Resource Book on Mental Health, Human Rights and Legislation. Geneva: World Health Organization, 2005. Available from: https://www.google.com/url?sa=t\&rct=i\&q=\&esrc=s\&so urce=web\&cd=\&ved=2ahUKEwi9kvehrpXsAhXOcnOKH YcrCPAQFjAAegQ|BBAC\&url=https\%3A\%2F\%2Fec.eu ropa.eu $\% 2$ Fhealth $\% 2$ Fsites $\% 2$ Fhealth $\% 2$ Ffiles $\% 2 F m e$ ntal_health\%2Fdocs $\% 2 F$ who_resource_book_en.pdf\& usg=AOvVaw14VhRAiKWnQMPofR2lfio-

4. Baillie L. Patient dignity in an acute hospital setting: A case study. Int J Nurs Stud. 2009 Jan;46(1):23-36. [PubMed] DOI: 10.1016/j.jinurstu.2008.08.003

5. Blomberg AC, Bisholt B, Lindwall L. Responsibility for patient care in perioperative practice. Nursing Open. 2018;5:414-421. [PubMed] DOI: 10.1002/nop2.153

6. Department of Health. Final report on the review of the Department of Health Dignity in Care Campaign. November 2009. [Accessed 7 September 2020]; Available

from: https://www.dignityincare.org.uk/_assets/Opinion_Lead er_Final_Report_to_DH.doc.pdf

7. Baillie L, llott L. Promoting the dignity of patients in perioperative practice. J Perioper Pract. 2010;20:27882. [PubMed] DOI: $10.1177 / 17504589100200080$ 